\title{
Effects of diosmine-hesperidine on experimental colonic anastomosis
}

\author{
Cengiz $\mathrm{A}^{1}$, Tabak $\mathrm{S}^{2}$, Kuru $\mathrm{S}^{1}$, Demirel $\mathrm{AH}^{1}$, Barlas $\mathrm{AM}^{1}$, Altun $\mathrm{T}^{3}$, Gonultas $\mathrm{M}^{4}$, Celepli $\mathrm{S}^{1}$, \\ Kilicoglu SS ${ }^{5}$, Aydogan $\mathrm{A}^{1}$, Kilicoglu $\mathrm{B}^{1}$
}

General Surgery Clinics of Training and Research Hospital of Ankara, Ulucanlar Altindag Ankara Turkey. kilicoglubulent@yahoo.com

\begin{abstract}
Aim: Our goal was to determine the effects of a diosmine-hesperidine combination on wound healing in a rat model of colonic anastomosis.

Materials and methods: In this study, 20 Wistar Albino female rats were randomized into four experimental groups containing five rats in each group. A segment of $1 \mathrm{~cm}$ of colon was excised $4 \mathrm{~cm}$ proximally to the peritoneal reflection in all rats without carrying out any mechanical or antibacterial bowel preparation. Colonic anastomosis was performed with interrupted, inverting sutures of $6 / 0$ polypropylene. Beginning from the first postoperative day, the rats in Groups II and IV received $100 \mathrm{mg} / \mathrm{kg}$ per day of diosmine-hesperidine via orogastic route by $4 \mathrm{~F}$ fine feeding catheter.

Results: A significant difference was detected between groups in terms of their hydroxyproline levels $(p<0.05)$; the hydroxyproline level of Group I was significantly lower than that of the other groups while no significant difference was noted between Groups II and III.

Conclusion: The administration of diosmine-hesperidine increased the amount of collagen and bursting pressures at the anastomotic site and thus had favorable influences on the healing of colonic anastomosis (Tab. 1, Fig. 3, Ref. 33). Full Text in PDF www.elis.sk.

Key words: diosmine-hesperidine, colonic anastomosis.
\end{abstract}

Colonic anastomosis remains a significant issue as a major cause of postoperative mortality and morbidity in colorectal surgery, and is associated with $25-37 \%$ of deaths according to the related literature review (1).

The segment in the gastrointestinal system at highest risk of anastomotic leakage is the colon, with an even higher risk in the distal colon $(2,3)$. It has been reported that $80 \%$ of all postoperative mortalities are associated with peritonitis, abscesses and sepsis secondary to anastomotic leak $(4,5)$. A lack of collateral connections for arterial vascularization and rich bacterial flora are associated with leaks during the postoperative period $(6,7)$. Moreover, concomitant medical conditions make colorectal surgery more risky for the patients undergoing colonic surgery $(8,9)$.

The main factors affecting the healing of colonic anastomosis include the applied surgical technique, oxygenation and vascularization of the anastomotic line, suture materials, blood transfusion, radiation therapy, mechanical bowel preparation, concomitant diseases, drug therapies, nutrition, age, adequate drainage, antibiotic administration and anastomotic tension $(10,11)$.

${ }^{1}$ General Surgery Clinics of Training and Research Hospital of Ankara, ${ }^{2}$ Obstetrics \& Gynecology Clinic, Medical Faculty of Adiyaman University, ${ }^{3} \mathrm{Obstetrics} \&$ Gynecology Clinic, Etlik Women's Health \& Maternity Hospital, ${ }^{4}$ Pathology Clinic, Training and Research Hospital of Ankara, and ${ }^{5}$ Histology-Embryology, Ufuk University Medical Faculty

Address for correspondence: B. Kilicoglu, Ankara Training \& Research Hospital General Surgery Department Ulucanlar Altindag, Ankara, Turkey. Phone: +905334222049
Flavonoids, also referred to as bio-flavonoids, are polyphenol antioxidants found naturally in plants. They are a large group of plant pigments sharing the same basic chemical structure; a threeringed molecule with hydroxyl $(\mathrm{OH})$ groups attached. In addition to their antioxidant activities, flavonoids also have many healthpromoting effects. Flavonoids also have antiallergic, anticancer, antioxidant, anti-inflammatory and antiviral activities $(12,13)$. The most important dietary sources are fruits, green and black tea, and soybean. Most of the bio-flavonoids are the yellow pigments found in citrus fruit as well as in other fruits and vegetables. The major bio-flavonoids found in citrus include diosmine, hesperidine, rutin, naringin, tangeretin, diosmetin, narirutin, neohesperidin, nobiletin and quercetin.

A micronized flavonoid fraction combination (90\% diosmine and $10 \%$ hesperidine) improves wound healing by fighting against inflammatory mediators and by maintaining microcirculation through its favorable effects on blood viscosity, thereby increasing the venous tonus and lymphatic drainage. We have determined the effects of a diosmine-hesperidine combination on wound healing in a rat model of colonic anastomosis.

\section{Materials and methods}

For all the experiments, female Wistar Albino rats with a mean weight of $265 \mathrm{~g}$ (240-290) were used. All rats were caged in the animal laboratory seven days before undergoing surgery. All rats were kept in separate cages at room temperature $\left(21^{\circ} \mathrm{C}\right)$, both 
before and after surgery. All animals had free access to water and standard feeding during the experiment. All experimental procedures were performed according to our institutions guide for the care and use of laboratory animals. The study was approved by the Ethical Committee at the Training and Research Hospital of Ankara on 06.12.2006 with issue number 1458.

Twenty female Wistar Albino rats were divided into 4 groups consisting of 5 rats in each. In Group I, all rats underwent colonic anastomosis. They did not receive any medical therapy. All rats were sacrificed on the third postoperative day to measure the colonic bursting pressure, determine the hydroxyproline level, and carry out the histopathological examination. This group was the control group on the third day. In Group II, $100 \mathrm{mg} / \mathrm{kg} / \mathrm{day}$ of diosmine-hesperidine were administered via orogastric route by $4 \mathrm{~F}$ fine feeding catheter on the first post-operative day following the colonic anastomosis. All rats were sacrificed on the third postoperative day to measure the colonic bursting pressure, determine the hydroxyproline level, and carry out the histopathological examination. This group was the treatment group on the third day. In Group III, all of the rats underwent colonic anastomosis. They did not receive any medical therapy. All of the rats were sacrificed on the seventh postoperative day to measure the colonic bursting pressure, determine the hydroxyproline level, and carry out the histopathological examination. This group was the control group on the seventh day. In Group IV, the rats were administered 100 $\mathrm{mg} / \mathrm{kg} /$ day of diosmine-hesperidine via nasogastric route on the first post-operative day following the colonic anastomosis. All rats were sacrificed on the seventh postoperative day to measure the colonic bursting pressure, determine the hydroxyproline level, and carry out the histopathological examination. This group was the treatment group on the seventh day.

\section{Surgical procedure}

The rats were randomly divided into 4 groups (Group I, Group II, Group III, and Group IV). No mechanical or antibacterial bowel preparation was performed. Following a six-hour fasting, the rats were anesthetized by administering $30 \mathrm{mg} / \mathrm{kg}$ of ketamine hydrochloride (Ketalar; Eczacibasi, Istanbul, Turkey). After abdominal shaving and cleaning the area by povidone-iodine solution, a median laparotomy was performed via an incision of $3 \mathrm{~cm}$ under sterile conditions. A segment of $1 \mathrm{~cm}$ of colon was excised $4 \mathrm{~cm}$ proximal to the peritoneal reflection in all rats. Anastomosis of the colon was performed with interrupted, inverting sutures of 6/0 polypropylene (Prolene; Ethicon, Somerville, NJ, USA). The abdominal fascia and skin were closed with continuous sutures of 4/0 silk (Ethicon). Post-operatively, $5 \mathrm{~mL}$ of saline solution was injected subcutaneously to prevent dehydration. All procedures were carried out by the same surgeon.

Rats in the treatment groups (Groups II and IV) were administered $100 \mathrm{mg} / \mathrm{kg}$ /day of diosmine-hesperidine on the first postoperative day via orogastric route by a $4 \mathrm{~F}$ fine feeding catheter.

\section{Measurement of the Bursting Pressure}

Bursting pressure measurement was carried out on the third postoperative day for the rats in Groups I and II, and on the sev- enth postoperative day for the rats in Groups III and IV. A colonic segment of $6 \mathrm{~cm}$ medial to the anastomotic line was resected following laparotomy after anesthesia with $30 \mathrm{mg} / \mathrm{kg}$ of ketamine hydrochloride. During the resection, in order to prevent injury in the anastomotic line, adhesions and the adjacent organs were simultaneously removed when indicated. The bursting pressures were measured before the removal of adjacent organs. The colon was washed with saline solution to eliminate the fecal content prior to bursting pressure measurement. Bursting pressure measurements were detected by using a Vernier Lab Pro and BPT 300 Pressure Transducer (May, Biopac Systems Inc, Commat Ltd., Ankara, Turkey).

A pressure transducer was connected to one end of a threeway valve; a $50 \mathrm{cc}$ injector was connected to the other end. The injector was operated in conjunction with an infusion pump (Secura Perfusor FTB, Braun) to maintain a stable infusion rate. The third end of the three-way valve was attached to the colonic segment, resected by a catheter, with double sutures of $2 / 0$ silk. The information received from the pressure transducer was transferred to a computer and the pressure alterations were recorded graphically using Logger Pro software. The system was calibrated by accepting the value obtained by keeping the valve leading to the colon open without air infusion as zero. The valve to the colon was then clamped and the infusion pump was switched on. The procedure continued until a pressure of $400 \mathrm{mmHg}$ was obtained on the monitor to test the system against leaks.

Bursting pressure measurements were performed successfully. The site of the burst was noted to determine whether it was at the site of the repair or in another segment.

\section{Determination of the tissue hydroxyproline level}

The colorimetric reaction, in which 4-hydroxyproline reacts with chloramine- $\mathrm{T}$ (N-chloro-p-toluenesulfonamide) to produce pyrrole or pyrrol-2-carboxylic acid and chromogen complex is produced by the reaction of $\mathrm{p}$-dimethylaminobenzaldehyde with its reagent prepared by perchloric acid (Erlich's reagent) after the oxidation of pyrrole or pyrrol-2-carboxylic acid is based on the spectrophotometric measurement of the chromogen complex at an absorbance of $560 \mathrm{~nm}$.

A segment of $1 \mathrm{~cm}$, including the site of the anastomosis, was resected and kept in a deep freezer at $-40{ }^{\circ} \mathrm{C}$ for the measurement of peri-anastomotic tissue hydroxyproline concentration, which is another indicative parameter of wound healing. The tissues were washed with saline solution and after the excess moisture had been removed by using filter paper, samples of $30 \mathrm{mg}$ were prepared for the measurement of hydroxyproline concentration. The tissue samples were placed in tubes containing $1 \mathrm{~mL}$ of $50 \mathrm{mM}$ potassium phosphate buffer $(\mathrm{pH}=7.0)$ and concentrated HCL of the same volume. Then, the $\mathrm{pH}$ value of the acidic solutions occurred upon adjusting the hydrolysis to $8-8.5$ by using $\mathrm{NaOH}$ and HCL solutions of various concentrations and phenolphthalein as an indicator.

One $\mathrm{mL}$ of $\mathrm{pH}$-adjusted hydrolysate was put into test tubes and after adding $2 \mathrm{~mL}$ of isopropanol and $1 \mathrm{~mL}$ of chloramine- $\mathrm{T}$ oxidant solution, they were mixed. The tubes were kept at room temperature $\left(17-21^{\circ} \mathrm{C}\right)$ for four minutes. Then, $13 \mathrm{~mL}$ of Ehrlich's 
692-697

reagent was added to the tubes and mixed. The tubes were closed by glass balls and incubated in water bath at $60^{\circ} \mathrm{C}$ for 25 minutes. After incubation, the tubes were being cooled under running tap water for 2-3 minutes. The absorbance was determined at 560 $\mathrm{nm}$ against a reagent blank and evaluated with respect to $40 \mu \mathrm{g}$ standard L-hydroxyproline solution.

\section{Histopathological examination}

After removing the adhesions. the colonic segments were kept in a $10 \%$ neutral formaldehyde solution at $+4{ }^{\circ} \mathrm{C}$. After obtaining sections of $5 \mu \mathrm{m}$ from paraffin blocks and staining them with hematoxylin-eosin dye, the sections were examined according to Verhofstadt Wound Scoring under a light microscope by a single physician,

\section{Statistical analysis}

The analysis of the data was performed using SPSS 10.0 software. While descriptive statistics for bursting pressure and hydroxyproline were expressed as the mean standard deviation, histopathological scores were expressed as the median (minimummaximum). The differences among groups with respect to bursting pressures and hydroxyproline levels were tested by using one-way analysis of variance (ANOVA). When the difference was significant, the post hoc Tukey test was used to establish the origin of the difference. The Kruskal-Wallis test was used to compare the histopathological scores of the groups. If the difference was significant, the Kruskal-Wallis nonparametric multiple comparison test was used to determine the origin of the difference. A p value less than 0.05 was considered statistically significant.

\section{Results}

\section{Assessment of tissue hydroxyproline levels}

A one-way ANOVA test was used to compare the hydroxyproline levels between the groups. A Tukey test was used to determine the origin of the differences. The results revealed that the hydroxyproline levels in Group I were significantly lower than in the other groups $(p<0.05)$, whereas no differences were detected between Groups II and III. Additionally, when Groups II and III were compared with Group IV, the hydroxyproline level in Group IV was found significantly higher than in Groups II and III (Fig. 1).

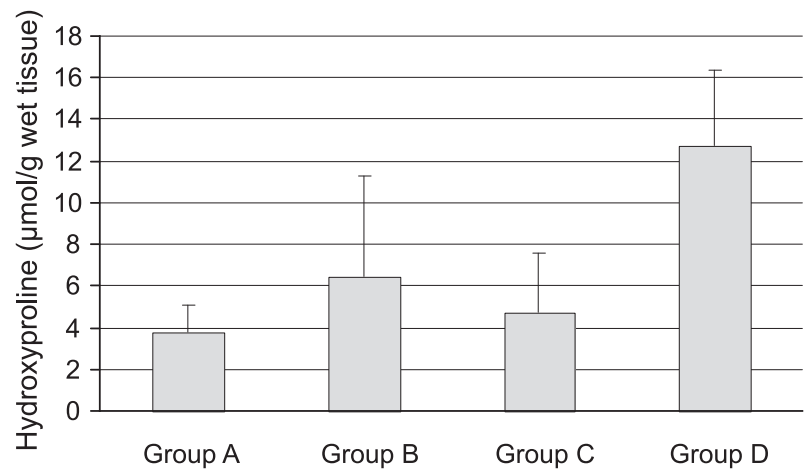

Fig. 1. Comparison of hydroxyproline levels between the groups.

\section{Assessment of the histological examination}

SPSS for Windows 10.0 software was used to perform statistical comparisons of histological examinations with the Kruskal-Wallis test for the evaluation of groups as a whole and the Mann-Whitney U test for the evaluation of groups individually. A statistically significant difference was only observed between Groups I and III in terms of macrophages ( $<<0.05$ ), while the other groups and values did not have any significant differences (Fig. 2).

\section{Assessment of colon bursting pressure}

When the bursting pressure values of the groups were compared, Group IV was noted to have the highest value. A statistically significant difference was observed between Groups I and II $(\mathrm{p}=0.05)$. The mean bursting pressure value in Group II was higher than that in Group I. There was also a statistically significant difference between Groups I and III ( $p=0.005)$, with Group III having higher bursting pressure values. The difference between Groups II and III was also statistically significant $(\mathrm{p}=0.005)$. Similarly, Groups III and IV as well as Groups II and IV were noted to have significant differences $(\mathrm{p}<0.05)$. The bursting pressures in Group IV were higher than noted in Groups II and III (Tab. 1 and Fig. 3).

\section{Discussion}

Anastomosis is one of the fundamental surgical procedures involving the gastrointestinal system, especially intraabdominal surgery. Therefore, anastomosis and its reliability are of critical

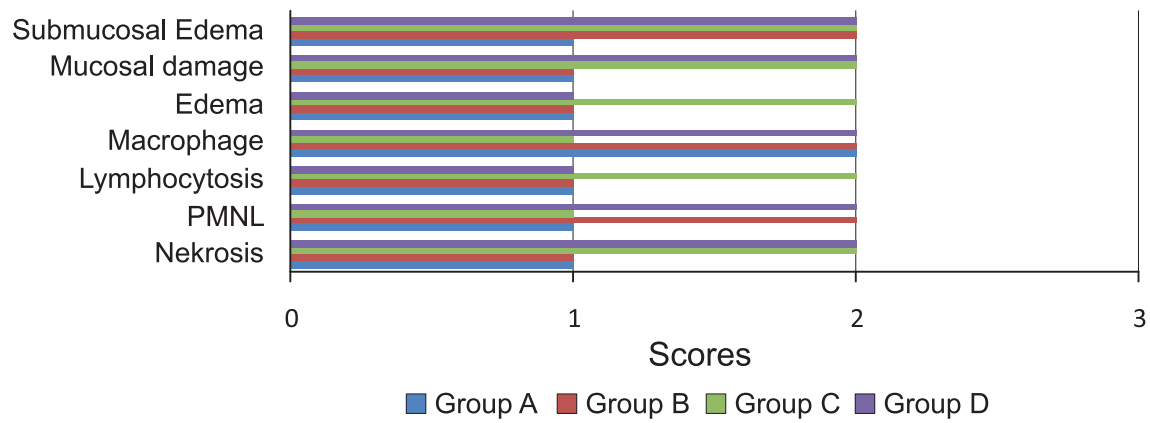

Fig. 2. Comparison of histopathologic data between the groups. 
Tab. 1. Mean and median values of bursting pressures in the groups.

\begin{tabular}{|c|c|c|c|c|}
\hline Groups (n) & $\begin{array}{c}\text { Group I } \\
(\mathrm{n}=5)\end{array}$ & $\begin{array}{l}\text { Group II } \\
\quad(n=5)\end{array}$ & $\begin{array}{c}\text { Group III } \\
(n=5)\end{array}$ & $\begin{array}{c}\text { Group IV } \\
(n=5)\end{array}$ \\
\hline Mean Pressure (mmHg) & 123.89 & 169.00 & 225.33 & 327.33 \\
\hline Median Pressure (mmHg) & 125.00 & 180.00 & 231.00 & 328.00 \\
\hline Minimum Pressure (mmHg) & 86.00 & 135.00 & 196.00 & 279.00 \\
\hline Maximum Pressure $(\mathrm{mmHg})$ & 175.00 & 240.00 & 250.00 & 354.00 \\
\hline
\end{tabular}

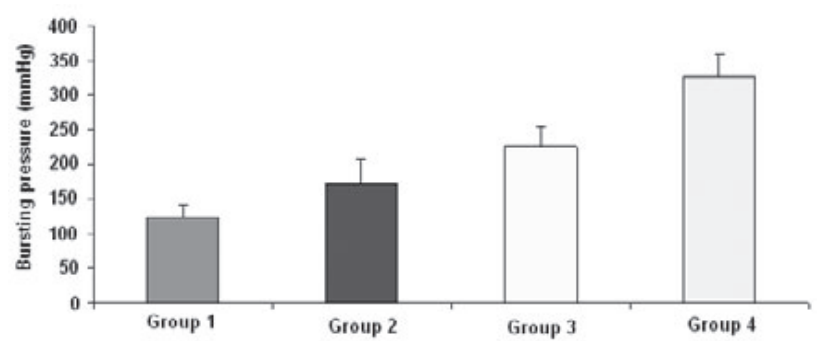

Fig. 3. Mean anastomosis bursting pressure according to the groups.

importance for surgeons. Clinical manifestations of anastomotic insufficiency usually occur during the first postoperative week. This fact underlines the importance of applying experimental methods of anastomotic healing that detect early changes in the anastomoses (14). Many factors affect the healing of colonic anastomoses. The factors which affect the healing of colonic anastomoses can be classified in two groups; the factors with an impact on collagen synthesis and factors associated with the surgery. Chronic malnutrition, diabetes, uremia, trauma, radiation damage, and older age are some of the factors with a negative impact on collagen synthesis. Factors associated with the surgery include tissue damage, infected tissue, and poor vascularization in the tissue or impaired tissue nutrition. Although the experience of the surgeon can affect the success of surgical process, the anastomotic site remains to be the limiting factor with the presence of factors impairing the collagen synthesis.

It has been reported that contrasting with endoscopy and gastrografin in patients undergoing colonic anastomosis demonstrates a high incidence of anastomotic leakage. If the leakage is minimal, it does not cause any postoperative morbidity, mortality or clinical manifestations (15).

Wound healing is regulated by locally produced mitogens and chemotactic factors. The process contains three major phases; migration of inflammatory cells towards the wound site and fibroblasis, formation of a new extracellular matrix and granulose tissue with microvascularization and the proliferation of natural cells of the tissue.

Diosmine-hesperidine is a drug used for patients with venous system diseases such as lower extremity venous insufficiency and hemorrhoids. It is known to increase the venous tone, regulate the capillary resistance and capillary permeability, as well as to increase the lymphatic drainage. There are also studies reporting the free-radical scavenging and leukocyte adherence-inhibiting effects of diosmine-hesperidine.

Diosmine-hesperidine has been shown, both in vitro and in vivo to inhibit the complement system activation (16). The mechanism of this effect has been proposed to be one of the antiinflammatory effects of the drug potentially contributing to ulcer healing. Korthuis et al (17) induced intestinal ischemia-reperfusion injuries in rat intestines and demonstrated that diosmine-hesperidine significantly decreased the ischemia-reperfusion injury in the intestines. Hasanoglu et al (18) treated the infected wounds of guinea pigs with systemic and topical diosmine-hesperidine, and reported beneficial results in wound healing. In a meta-analysis study conducted by Ramelet et al (19), diosmine-hesperidine administration as an adjuvant therapy to conventional therapy was demonstrated to be more effective than conventional therapy alone on ulcer-associated symptoms and accelerating ulcer healing in patients with venous ulcers induced by chronic venous insufficiency. Similarly, in a study conducted by Pecking et al (20) on the effects of diosminehesperidine in upper extremity lymphedema occurring after breast cancer operation, the positive effects of diosmine-hesperidine on the treatment of lymphedema by accelerating the lymphatic flow, decreasing the intralymphatic pressure significantly, and also by accelerating the reflow of interstitial fluid to capillaries were reported, Tanaka et al (21) demonstrated the chemoprotective effects of naturally occurring flavonoids (e.g., grapefruit) on carcinogenesis and maintained that the particular effect of flavonoids was mediated by inhibiting the increased cell proliferation. There are other studies which have reported the chemoprotective effects of flavonoids (22). Kilicoglu S et al have used the agent and the beneficial effects of propolis on wound healing of colon anastomosis (23). Gollu et al investigated the effects of honey on colon anastomose healing and preventing the ntraabdominal adhesions (24).

Healing in intestinal anastomosis can be evaluated mechanically, biochemically, or histopathologically. While the bursting pressure or breaking strength is taken into consideration for mechanical evaluation, the rate, quantity and characteristics of collagen production at the anastomosis site are considered in the biochemical evaluation. Independently of other intestinal wall events (age and weight-adjusted standard hydroxyproline level per tissue) the produced collagen content localized in the anastomosis site after the third day is a reliable biochemical indicator of anastomosis (5).

The present study was designed to describe the effect of diosmine-hesperidine on wound healing in rat following colonic anastomosis. Anastomosis bursting pressures, peri-anastomotic tissue hydroxyproline levels, and histopathology of peri-anastomotic tissue were evaluated for this purpose.

Although significant differences were established in terms of anastomosis bursting pressures and tissue hydroxyproline determination parameters, the histopathological examination did not reveal any significant differences. 
692-697

One of the main results obtained in the present study was the increase in bursting pressures as a result of diosmine-hesperidine administration and consequently, improved the anastomotic strength. In the study conducted by Cronin (25), it was reported that the anastomotic strength applied after the third postoperative day gradually increased to its maximum levels in the period from seventh to tenth day. Jilborn (26) maintained that bursting could be expected in a location other than in the anastomosis site after the seventh postoperative day. Therefore, anastomotic bursting pressure was evaluated on the third and seventh days. Breaking strength, which is another parameter of assessment suggested by Hendriks (5), was not evaluated in our study as being a marker for wound healing at a later stage, and retrieving slowly. The comparison of bursting pressures demonstrated that the highest values were obtained in Group IV, which had been administered diosmine-hesperidine for three days prior to anastomosis and had undergone bursting pressure evaluation on the seventh postoperative day. There was a significant difference between the values in Groups I and II ( $\mathrm{p}=0.005)$. Group II had higher bursting pressure values than Group I. The differences between Groups I and III were also significant; Group III had higher values than the Group I. A significant difference was also established between Groups II and III in terms of bursting pressures $(\mathrm{p}=0.005)$. Similarly, there were statistically significant differences between Groups III and IV, as well as between Groups II and IV ( $\mathrm{p}<0.001)$. The bursting pressures in Group IV were higher than those observed in Groups II and III. Anastomotic site bursting pressure is one of the most critical markers in assessing the anastomotic strength and integrity during healing (5). Bearing in mind the fact that sutures provide strength of the anastomotic line during the early stages in which the collagen concentrations decrease, a direct association between the suture technique and resistance to pressure has been proposed $(27,28)$. Using the same suture technique and material, and performing anastomosis by the same surgeon allowed evaluating the diosmine-hesperidine administration as an independent factor in the present study.

Clearly, the suture technique is not the only determinant of tensile strength. Often, the most significant indicator of breaking strength is the collagen accumulation in the tissues (29). Another important finding in the present study was the increase in tissue hydroxyproline levels obtained as a result of diosmine-hesperidine administration. When evaluating the tissue hydroxyproline levels, age and weight-adjusted hydroxyproline level per tissue were also considered. This is defined as hydroxyproline content and is a parameter independent of other intestinal wall events.

Free oxygen radicals produced during ischemic events impair the wound healing by inhibiting the contraction of the collagen matrix (30). In addition to free oxygen radicals, cytokines have also a negative impact on wound healing. Cytokines inhibit the TNF- $\alpha$ collagen production and increase the collagen breakdown (31). These mediators released during reperfusion injury may impair the wound or anastomoses healing (32). Duchene-Marullaz et al (33) demonstrated that administration of diosmine-hesperidine significantly decreased the free radical activity, and chemotactic reaction induced by leukocyte phagocytosis and free radicals.
Another critical stage in the present study investigating the effects of diosmine-hesperidine administration on colonic anastomoses was the histopathological examination. Verhofstadt Wound Scoring was used for the scoring procedure. All pathologic specimens were evaluated by the same pathologist who was blinded to the study. Only Groups I and III differed significantly in terms of macrophages $(p<0.05)$, while the differences between the other groups and values were not significant; this particular result did not make any significant contribution to our conclusions. Histopathological examination is a more visual method when compared with bursting pressure measurement and tissue hydroxyproline level determination. It must be kept in mind that the pathologist is a factor that also must be considered. Thus, we maintain that bursting pressure measurement and tissue hydroxyproline level determination are more valuable parameters in the evaluation of healing of colon anastomoses. Many studies have only assessed the tissue hydroxyproline level and bursting pressure measurements in the evaluation of colonic anastomoses.

As a result, considered collectively, this present study and literature findings suggest that the administration of diosminehesperidine increases the amount of collagen and bursting pressure at the anastomosis site and positively influences the healing of colonic anastomoses.

\section{References}

1. Kodner IR, Fry RD. Colon, Rectum and Anus. Principles of Surgery. Schwartz SI (Ed). New York: Mc Graw-Hill, 1999, p. 1265-1283.

2. Fielding LP, Steward-Browns, Kearney G. Anastomotic integrity after operation for large bowel cancer: a multicentre study. BMJ 1980; 281: 411-414.

3. Matheson NA, Irving AD. Single layer anastomosis in the gastrointestinal tract. Surg Gynecol Obstect 1976; 143: 619-624.

4. Duncan JP, Chong S, Eric W et al. Effects of chronic corticosteroid and vitamin A on healing of intestinal anastomoses. Am J Surg 1992; 163: 72-77.

5. Hendriks T, Mastboom VJ. Healing of experimental intestinal anastomoses parameters for repair. Dis Colon Rectum 1990; 33: 890-901.

6. Hongstrom H, Hanglud U, Zedelfeld B. Tension leads increased neutrophil accumulation and decreased laparatomy wound strength. Surgery 1999; 107: 215-218.

7. Uluocak K. Kolon anastomoz yetersizliklerinin sebepleri ve önlenmesi. Dirim 19. 1992; 67: 34-42.

8. Beahs OH. Complications of colonic surgery. Surg Clin North Am 1967; 47: 983.

9. Schrock TR, Denevey JW, Dumphy JE. Factors contributing to leakage of colonic anastomoses. Ann Surg 1973: 177: 513-518.

10. Thornton FJ, Barbul A. Healing in the gastrointestinal tract. Surg Clin Nort Am 1997; 77 (3): 549-573.

11. Karahasanoglu T, Altinli E, Hamzaoglu I, Paksoy M, Yesildere T, Alemdaroglu K. Effect of growth hormone treatment on the healing of the left colonic anastomoses protein-malnourished rats. Br J Surg 1998; 85 (7): 931-933.

12. Feredioon S, Janita PK, Wanasundara PD. Phenoloic antioxidants. Crit Rev Food Sci Nutrit 1992; 32 (1): 67-103. 
13. Zabel DD, Hunt TK, Mueller RV, Goodson WH. Wound healing, Current Surgical Diagnosis and treatment. 11th edition. Way LW, Doherty GM (Eds). Lange Medical Books Mc Graw-Hill 2003; 86-99.

14. Karanjia ND, Corder AP, Holdsworth PJ, Heald RJ. Risk of Peritonitis and Fatal Septicemia and need to Defunction the Low Anastomosis. Br J Surg 1991; 78: 196-198.

15. Choti MA. Obstruction of large bowel. Curr Surg Ther. Cameron J L (Ed). St. Louis: Mosby-Year Book, Inc., 1995, 162 p.

16. Di Perri T, Auteri A. Action of S5682 on the complement system (in vitro and in vivo study). Inter Anjio 1988; 7 (2): 11-15.

17. Korthius RJ, Gute DC. Postischemic leukocyte/ endothelial cell interactions and microvascular barrier dysfunction in skeletal muscle: Cellular mechanism and effect of Daflon $500 \mathrm{mg}$. Int J Microcirc Clin Exp 1997; 17 (1): 11-17.

18. Hasanoglu A, Ara C, Ozen S, Kali K, Senol M, Ertas E. Efficacy of micronized Flavonoid fraction in healing of clean and infected wounds. Int J Angiol 2001; 10 (1): 41-44.

19. Coloridge- Smith P, Lok C, Ramelet AA. Venous leg ulcera metaanalysis of adjunctive therapy with micronized purified flavonoid fraction. Eur J Vasc Endovasc Surg 2005; 30 (2): 198-208.

20. Pecking AP, Fevrier B, Wargon C, Pillion G. Efficacy of Daflon 500 $\mathrm{mg}$ in the treatment of lymphedema (secondary to conventional therapy of breast cancer). Angiology 1997; 48 (1): 93-98.

21. Tanaka T, Makita H, Kawabata K, Mori H, Kakumoto M, Satoh K, Hara A, Sumida T, Tanaka T, Ogawa H. Chemoprevention of azoxymethane-induced rat colon carcinogenesis by the naturally occurring flavonoids, diosmine and hesperidine. Carcinogenesis 1997; 18 (5): 957.

22. Yang M, Tanaka T, Hirose Y, Deguchi T, Mori H, Kawada Y. Chemopreventive effects of diosmine and hesperidine on N-butyl-N-(4-hydroxybutyl) nitrosamine-induced urinary-bladder carcinogenesis in male ICR mice. Int J Cancer 1997; 73 (5): 719.
23. Kilicoglu SS, Kilicoglu B, Erdemli E. Ultrastructural view of colon anastomosis under propolis effect by transmission electron microscopy. World J Gastroenterol 2008; 14 (30): 4763-4770.

24. Gollu A, Kismet K, Kilicoglu B, Erel S, Gonultas MA, Sunay AE, Akkus MA. Effect of honey on intestinal morphology, intraabdominal adhesions and anastomotic healing. Phytother Res 2008; 22 (9): 1243-1247.

25. Cronin K, Jackson DS, Dunphy JE. Changing bursting strength and collagen content of the healing colon. Surg Gynecol Obstet 1968; 126 (4): 747-753.

26. Jilborn H, Ahonen J, Zedeerfeldt B. Healing of experimental colonic anastomoses I. Bursting strength of the colon after left colon resection and anastomoses. Am J Surg 1987; 136: 587-594.

27. Koruda MJ, Rolandelli RH. Experimental studies on the healing of colonic anastomoses. J Surg Res 1990; 48: 504-515.

28. Jilborn H, Ahonen J, Zederfeldt B. Healing of experimental colonic anastomoses. Collagen metabolism in the colon after left colon resection. Am J Surg 1980; 139 (3): 398-405.

29. Shandall A, Lowndes R, Young HL. Colonic anastomotic healing and oxygen tension. Br J Surg 1958; 72 (8): 606-609.

30. Arisawa S, Arisawa T, Ohashi M, Nitta Y. Effect of the hydroxyl radical on fibroblast mediated collagen remodeling in vitro. Clin Exp Pharmacol Physiol 1996; 23: 222.

31. Mauviel A, Daireaux M, Redini F, Pujol J.P. Tumor necrosis factor inhibits collagen and fibronectin synthesis in human dermal fibroblasts. FEBS Lett 1998; 236: 47.

32. Kuzu MA, Koksoy C, Kale IT. Reperfusion injury delays healing of intestinal anastomoses in rat. Am J Surg 1998; 176: 348-351.

33. Duchene-Marullaz P, Duhault J. Interference of S 5862 with 2 types of free radical induced effects. X. The International Congress of Pharmacology-Sydney August 1987. 